\title{
What Is Clean Cities?
}

Clean Cities is a government-industry partnership designed to reduce petroleum consumption in the transportation sector by advancing the use of alternative fuels and vehicles, idle reduction technologies, hybrid electric vehicles, fuel blends, and fuel economy measures.

Sponsored by the U.S. Department of Energy's (DOE) Vehicle Technologies Program (VTP), Clean Cities contributes to the energy, environmental, and economic security of the United States by supporting local decisions to reduce our dependence on imported petroleum. Established in 1993 in response to the Energy Policy Act (EPAct) of 1992, the partnership provides tools and resources for voluntary, community-centered programs to reduce consumption of petroleum-based fuels.

In almost 90 coalitions, government agencies and private companies voluntarily come together under the umbrella of Clean Cities. The partnership helps all parties identify mutual interests and meet the objectives of reducing the use of imported oil, developing regional economic opportunities, and improving air quality.

\section{Which technologies are included?}

The portfolio provides a range of options and flexibility to meet the petroleum reduction goals.

Clean Cities deploys technologies and practices developed by VTP. These include truck stop electrification and onboard auxiliary power to reduce fuel used in idling trucks, hybrid electric vehicles, the blending of nonpetroleum based fuels (such as ethanol or biodiesel) with conventional fuels, higher efficiency vehicles and driving practices, and the cornerstone of the portfolio, alternative fuels. The alternative fuels, which are defined by EPAct and supported by Clean Cities, include ethanol, biodiesel, hydrogen, electricity, liquefied petroleum gas (propane), and natural gas.

\section{How does Clean Cities work?}

\section{The partnership mobilizes local stakeholders in} government and industry.

Clean Cities draws stakeholders from local, state, and federal agencies; public health and transportation departments; commercial fleets; transit agencies; and other government offices; as well as auto manufacturers, car dealers, fuel and equipment suppliers, public utilities, and nonprofit associations. More than 5,500 stakeholders have accepted the invitation to contribute to the partnership's mission.

Clean Cities is instrumental in cultivating an advanced transportation community in which people learn about a wide range of options and technologies. In addition, Clean Cities helps create markets for alternative fuel vehicles (AFVs), hybrids, fuel blends, fuel economy, and idle reduction. A Clean Cities coalition can provide a forum for members to leverage their resources, develop joint projects, collaborate on public policy issues, and promote petroleum reduction and clean air technologies.

The partnership addresses the challenge of moving the United States away from the infrastructure and practices that contribute to dependence on imported petroleum and toward energy independence and security. In support of this challenge, Clean Cities assists the nation in meeting its objectives for renewable and alternative fuel use.

\section{What kind of assistance does Clean Cities offer? Clean Cities provides coordinated strategies and technical and funding resources.}

At the national level, Clean Cities provides original equipment manufacturers, trade associations, and other federal agencies with coordinated strategies and resources they can leverage to obtain maximum petroleum reduction. Clean Cities also provides coalitions with access to information and incentives from DOE and other federal agencies and industry partners that can help fund significant, high-impact projects.

Additionally, Clean Cities provides a large collection of technical data and information, including current data on fuels, vehicles, fueling station and truck-stop electrification locations, infrastructure development, state and federal incentives and laws, technical and outreach publications, and industry resources-all available online. Finally, Clean Cities offers technical assistance to coordinators as they work to develop infrastructure and market strategies. 


\section{Contacts}

Clean Cities staff members are available to assist you.

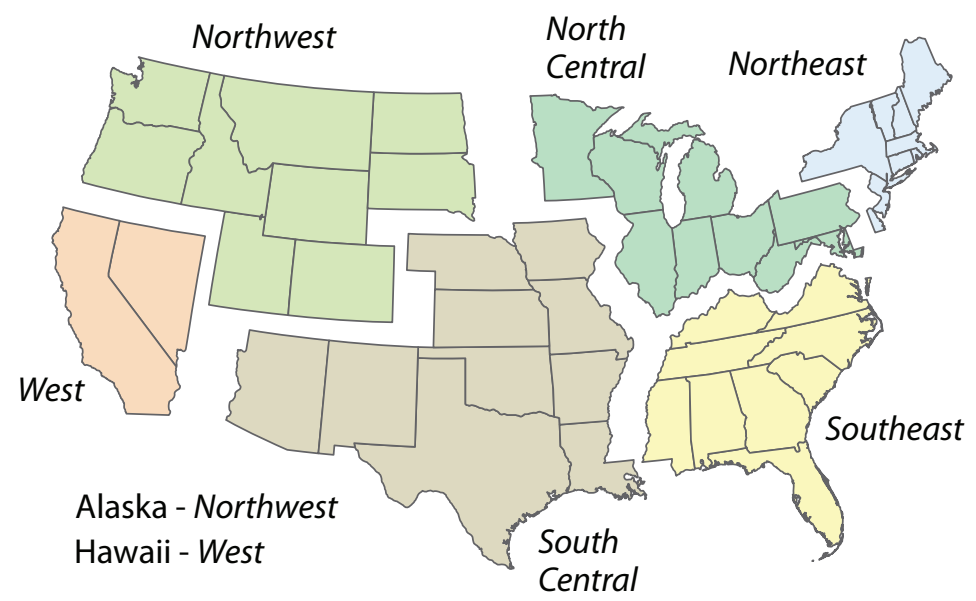

DOE Headquarters

Dennis A. Smith

Technology Deployment Manager and Clean Cities Director dennis.a.smith@ee.doe.gov 202-586-1791

\section{DOE Regional Managers}

Mike Scarpino

Northeast Region

michael.scarpino@netl.doe.gov

412-386-4726

Kay Kelly

North Central Region

kay.kelly@netl.doe.gov

304-285-4535

Steven Richardson

Southeast Region

steven.richardson@netl.doe.gov

304-285-4185
Linda Bluestein

Clean Cities Co-Director linda.bluestein@ee.doe.gov 202-586-6116
Neil Kirschner

South Central Region

neil.kirschner@netl.doe.gov

412-386-5793

Doug Seiter

Northwest Region

doug.seiter@go.doe.gov

303-275-4810

Mike Bednarz

West Region

michael.bednarz@netl.doe.gov 412-386-4862

\section{Clean Cities Coordinators}

\begin{abstract}
AR-Arkansas
Annett Pagan

501-280-3079

Corey Woods

602-258-7505, x17

www.cleanairaz.org

\section{AZ-Tucson}

Colleen Crowninshield

520-792-1093, x426

EnvironmentalPlanning/

aspx

Curtis Martin

661-265-6000

CA-Central Coast

(San Luis Obispo)

Melissa Guise

805-781-4667

www.c-5.org
\end{abstract}

AZ-Valley of the Sun (Phoenix)

www.pagnet.org/Programs/

CleanFuels/tabid/180/Default.

CA-Antelope Valley (Lancaster)

CA-Coachella Valley Region

Richard Cromwell III

760-329-6462

\section{CA-East Bay (Oakland) \\ Chris Ferrara}

415-973-5408

\section{CA-Long Beach}

Vivian Ozuna

562-570-5405

CA-Los Angeles

Heloise Froelich

213-978-0854

www.cityofla.org/EAD/EADWEb-

AQD/AlternativeFuelMobile.htm

\section{CA-Sacramento}

Jill Egbert

530-757-5235

www.cleancitiessacramento.org

CA-San Diego Region

Greg Newhouse

619-388-7673

www.sdcleanfuels.org
CA-San Francisco

Vandana Bali

415-355-3728

CA-San Joaquin Valley

(Bakersfield)

Linda Urata

661-835-8665

CA-Silicon Valley (San Jose)

Margo Sidener

408-998-5865

www.svcleancities.org/

CA-Southern California Assn. of Governments (Diamond Bar) JoAnn Armenta

909-396-5757

www.the-partnership.org/

ccities.htm

CA-Western Riverside County

Barbara Spoonhour

951-955-8313

www.wrcog.cog.ca.us

CO-Denver

Natalia Swalnick

303-847-0271

www.lungcolorado.org/

CleanCities.htm

CO-Northern Colorado

Robin Newbrey

970-689-4845

http://northcolocleancities.com

C0-Southern Colorado

Alicia Archibald

719-494-6592

http://southern.

cleancitiescolorado.org

CT-Capital Clean Cities

(Manchester)

Craig Peters

800-255-2631

CT-Southwestern Area

(Fairfield)

Ed Boman

203-256-3010
CT-New Haven

Lee Grannis

203-627-3715

www.nhcleancities.org

CT-Norwich

Peter Polubiatko

860-887-6964

www.norwichcleancities.org

DC-Washington

George L. Nichols

202-962-3355

DE-State of Delaware

Suzanne Sebastian

302-735-3480

FL-Gold Coast (Miami/Fort

Lauderdale/West Palm Beach)

Larry Allen

954-985-4416

www.sfrpc.com/fgcccc.htm

FL-Space Coast (Orlando)

Bill Young

321-638-1443

www.clean-cities.org

\section{GA-Atlanta}

Wendy Morgan

678-858-5338

www.cte.tv/cca/cleancitiesatl.html

GA-Middle Georgia

Charise Stephens

478-803-2506

www.mga-cleancities.com

HI-Honolulu

Robert Primiano

808-768-3500

www.hawaii.gov/dbedt/ert/cc/

\section{IA-State of lowa}

Brian Crowe

515-725-2066

www.energy.iowa.gov/ICCC/

index.html

ID-Treasure Valley (Boise)

Beth Baird

208-384-3984

www.tvcleancities.org
IL-Chicago

Samantha Bingham

312-744-8096

www.chicagocleancities.org

IN-Central Indiana Clean

Cities Alliance

Kellie Walsh

317-985-4380

www.cicca.org

IN-South Shore (Gary)

Carl Lisek

219-365-4289

www.southshorecleancities.org

KS/MO-Kansas City

Kelly Gilbert

816-531-7283

www.kcenergy.org/

transportation.html

KY-Commonwealth Clean Cities Partnership (State of KY)

Melissa Howell

502-452-9152

www.kentuckycleanfuels.org

LA-Baton Rouge

Tammy Morgan

225-389-5491

www.gbrccc.org

LA-SE Louisiana Clean Fuels

Rebecca 0tte

504-568-6622

www.cleanfuelpartnership.org

MA-Massachusetts

David Rand

617-626-7338

www.mass.gov/doer

MD-State of Maryland

Chris Rice

410-260-7207

http://energy.maryland.gov/

incentives/transportation/

cleancities/index.asp
ME-Maine Clean Communities Steven Linnell

207-774-9891

www.gpcog.org/Transportation and_Land_Use/Maine_Clean Communities.php

MI-Ann Arbor

Sean Reed

888-818-0987

www.aacleancities.org

MI-Detroit

Pamela Hurtt

313-833-0100, x270

www.nextenergy.org/services/ collaborativeprograms/wg

cleancities.aspx

MI-Greater Lansing

Maggie Striz Calnin

517-925-8649, Ext. 3

www.michigancleancities.org

MN-Twin Cities

Lisa Thurstin

651-223-9568

www.cleanairchoice.org/cities

MO-St. Louis

Kevin Herdler

314-822-5831

www.stlcleancities.org

NC-Centralina Council of

Governments (Charlotte)

Jason Wager

704-348-2707

www.4cleanfuels.com

NC-Triangle Coalition (Raleigh, Durham, Chapel Hill)

Kathy Boyer

919-558-9400

www.trianglecleancities.org

ND-Red River Valley (Grand

Forks/Winnipeg, MB, Canada)

Valerie Kummer

800-252-6325

www.cleanairchoice.org/cities/ rrv.cfm 


\section{Geographical Coverage of Clean Cities Coalitions}

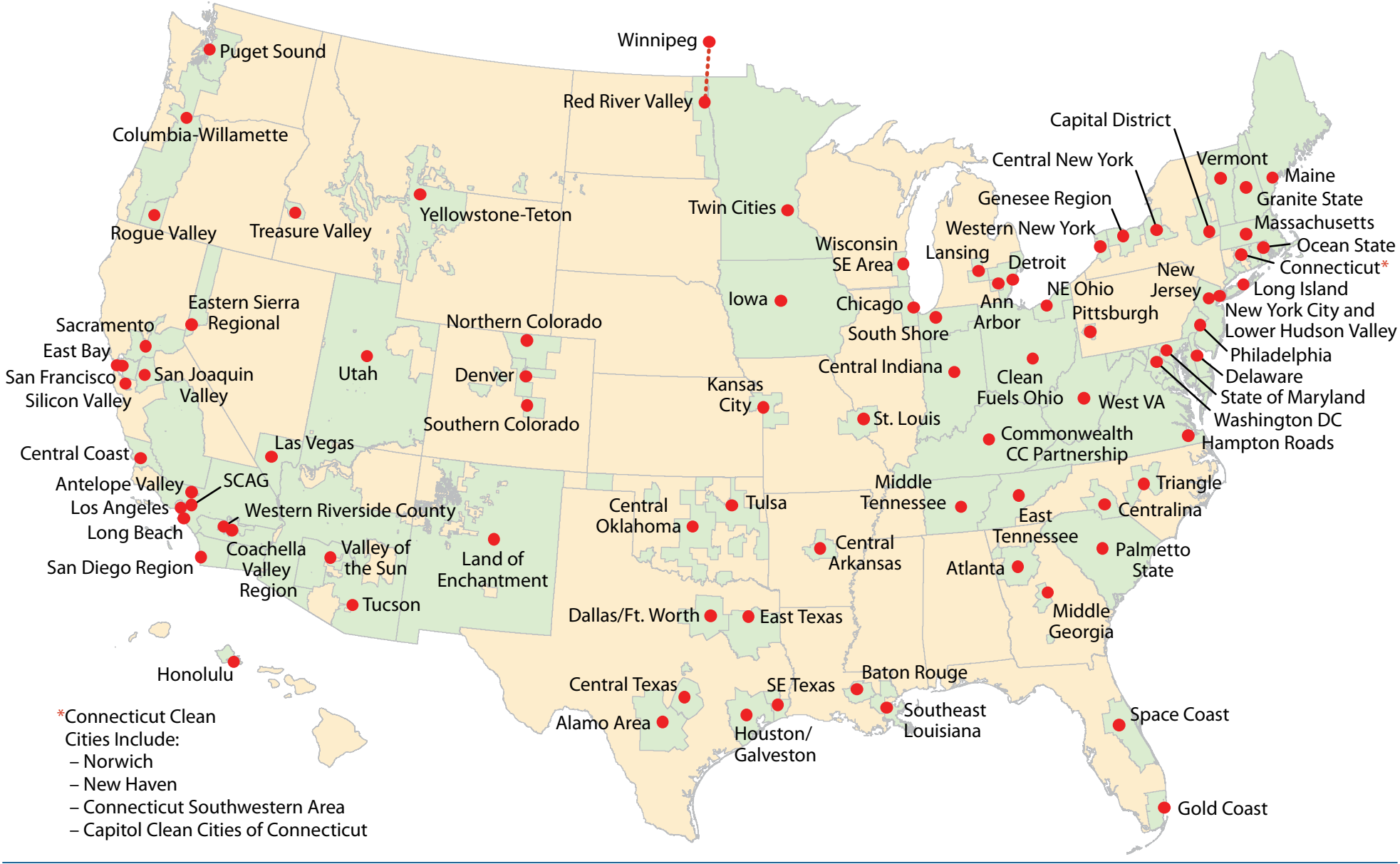

\section{NH-Granite State (State of NH)}

Barbara Bernstein

603-271-6751

www.granitestatecleancities. nh.gov/

\section{NJ-New Jersey}

Ray Willer

908-380-1109

NM-Land of Enchantment

(Albuquerque)

Frank Burcham

505-856-8585

NV-Las Vegas

Dan Hyde

702-229-6971

www.lasvegascleancities.org

NV-Eastern Sierra Regional

(Reno)

James Brandmueller

775-323-5879

NY-Capital District (Albany)

Deborah Stacey

518-458-2161

www.cdtcmpo.org/cdcc/cdcc.htm

\section{NY-Central New York}

(Syracuse)

Joseph Barry

315-498-2548

www.cc-cny.com

NY-Genesee Region (Rochester)

David Keefe

585-301-2433

www.grcc.us
NY-Long Island

Rita D. Ebert

631-969-3700 x25

www.gliccc.org

NY-New York City

Christina T. Ficicchia

212-487-4214

NY-Western New York (Buffalo)

Bill Pauly

716-435-5261

www.cleancommunitiesofwny.org

OH-Clean Fuels Ohio

(Columbus)

Sam Spofforth

614-884-7336

www.cleanfuelsohio.org

OH-Northeast Ohio (Cleveland)

John McGovern

216-281-6468 x223

www.earthdaycoalition.org/

cleantransport/

OK-Central Oklahoma

(Oklahoma City)

Yvonne Anderson

405-234-2264

www.okcleancities.com

OK-Tulsa

Nancy Graham

918-584-7526

www.tulsacleancities.com

For the most current list of Clean Cities coordinators, visit www.afdc.energy.gov/cleancities/progs/coordinators.php.

OR-Columbia-Willamette

(Salem)

Matt Hale

503-373-7560

www.cwcleancities.org

OR-Rogue Valley (Medford)

Sue Kupillas

541-245-0770

PA-Philadelphia

Dennis Winters

215-790-1894

www.phillycleancities.org

PA-Pittsburgh

Ryan Walsh

412-241-9323

www.cleancities-pittsburgh.org

RI-Ocean State (State of RI)

Wendy Lucht

401-874-2792

www.uri.edu/cels/ceoc/osccc.

html

SC-Palmetto State (State of SC)

Erika H. Myers

803-737-7951

www.palmettocleanfuels.org

TN-East Tennessee (Knoxville) Jonathan G. Overly

865-974-3625

http://eerc.ra.utk.edu/etcfc/index html
TN-Middle Tennessee

(Nashville)

Dave Pelton

615-482-4849

www.tennesseecleanfuels.org

TX-Alamo Area (San Antonio)

Andrew Hudgins

210-362-5228

www.aacog.com/cleancities/

default.asp

TX-Central Texas (Austin)

Stacy Neef

512-482-5343

www.ci.austin.tx.us/cleancities/

\section{TX-Dallas/Fort Worth}

Mindy Mize

817-608-2346

www.nctcog.org/trans/clean/

cities

TX-East Texas (Kilgore)

Rick McKnight

903-984-8641

www.netac.org/cities.htm

TX-Houston-Galveston

Christine M.B. Smith

832-681-2556

www.houston-cleancities.org

TX-South East Texas

(Beaumont)

Bob Dickinson

409-899-8444 x251

www.setccities.org
UT- Utah (Salt Lake City)

Robin Erickson

801-535-7736

www.utahcleancities.org

VA-Hampton Roads (Virginia)

Chelsea Jenkins

757-233-8982

www.hrccc.org

VT-State of Vermont

Karen Glitman

802-656-8868

www.uvm.edu/ cleancty

WA-Puget Sound (Seattle)

Stephanie Meyn

206-689-4055

www.pugetsoundcleancities.org

WI-Southeast Area

(Milwaukee)

Francis X. Vogel

414-221-4958

www.wicleancities.org

WV-State of West Virginia

Kelly Bragg

800-982-3386, x2004

www.energywv.org/community/ cleanstate.html

WY-Yellowstone-Teton

(Wyoming, Montana, Idaho)

Sandy Shuptrine

307-733-6371

www.yellowstonetetonclean

energy.org 


\section{What has Clean Cities accomplished?}

Since 1993, Clean Cities and its stakeholders have reduced petroleum consumption by 2 billion gallons.

Clean Cities is integral to many success stories. It has spurred significant gains in the nation's AFV population and associated fueling infrastructure. Clean Cities tracks the activities and accomplishments of its coalitions, translating them into petroleum reduction. Clean Cities stakeholders annually submit data regarding alternative fuel-blend sales, AFV and hybrid electric vehicle deployment, idle reduction initiatives, and fuel economy measures. Clean Cities then analyzes the data to identify equivalent petroleum fuel reduction impacts. This tracking represents only a small percentage of the actual impact of Clean Cities. The program lays the groundwork for local and regional partnerships to increase the use of alternative and advanced vehicles and fuels, which ripples out from the coalitions.

Through local and national events, special emphasis is focused on educating fleet managers and interested stakeholders about Clean Cities technologies. In addition, Clean Cities has created many funding opportunities through a variety of financial support programs, making available millions of dollars and leveraging millions more in categories such as fuel infrastructure, AFV incremental cost buy-downs, and idle reduction education.

\section{Where can I find more information?}

Visit the following DOE Web sites to find programmatic information and technical data.

- Clean Cities: This site features program background and accomplishments, an online newsletter, and a toolbox (www.eere.energy.gov/cleancities).

- Alternative Fuels and Advanced Vehicles Data Center (AFDC): This comprehensive site provides detailed information on Clean Cities' five portfolio areas and has searchable databases of more than 3,000 documents and incentives and laws ( $w w w . a f d c . e n e r g y$. gov/afdc). Also available on the AFDC:

- Vehicle Make/Model Search-Look up specs on alternative fuel and advanced technology vehicles and trucks and buses (www.afdc.energy.gov/afdc/progs/ vehicles_search.php).

- Alternative Fueling Station Locator-Find fueling sites offering alternative fuels across the country (www.afdc.energy.gov/afdc/fuels/stations_locator.html).

- FuelEconomy.gov: This site compares gas mileage, emissions, air pollution ratings, and safety data for new and used vehicles.

- EERE Information Center: This service provides assistance on energy efficiency and renewable energy topics. Inquiries requiring more technical expertise are forwarded to Clean Cities specialists (877-337-3463).

\section{Cumulative Petroleum Fuel Equivalents Reduced}

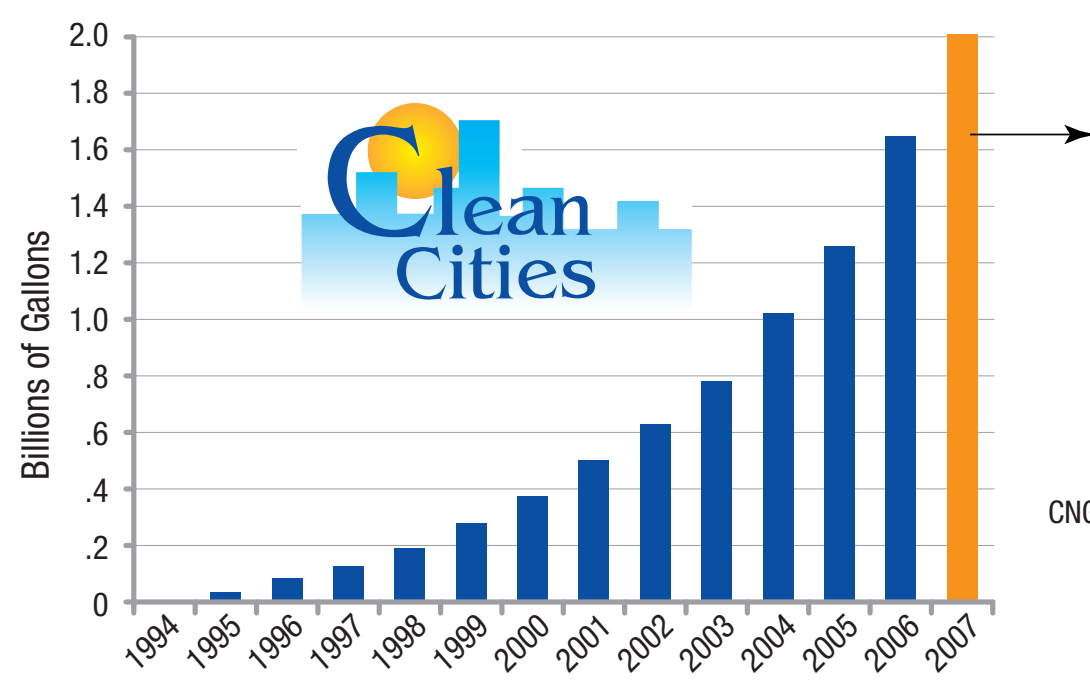

2007 Petroleum Reduction by Technology Type*

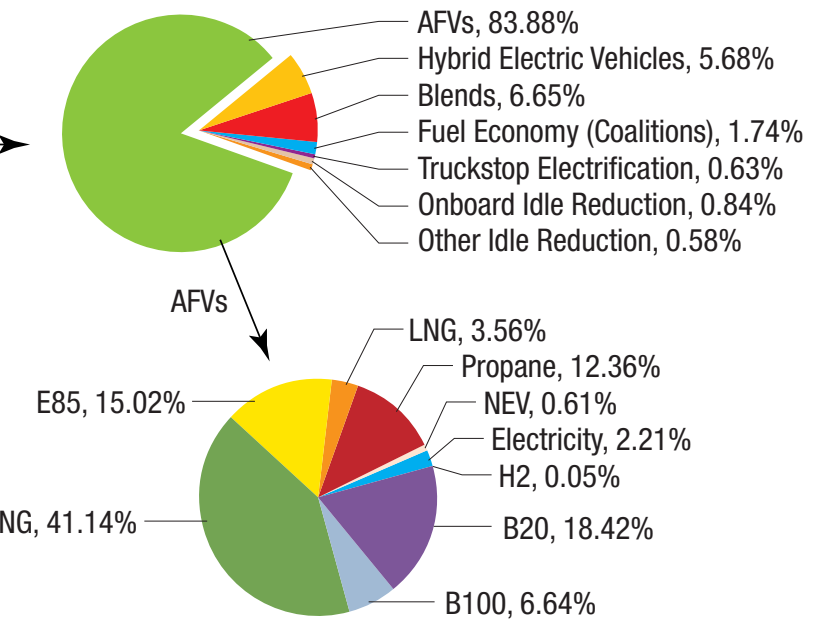

Breakdown of AFV Petroleum Reduction by Vehicle Type

* Latest information available as of April 1, 2009

Sponsored by the U.S. Department of Energy

Energy Efficiency and Renewable Energy

Vehicle Technologies Program

For more information contact: EERE Information Center 1-877-EERE-INF (1-877-337-3463) www.eere.energy.gov

Prepared by the National Renewable Energy Laboratory (NREL) NREL is a national laboratory of the U.S. Department of Energy Office of Energy Efficiency and Renewable Energy Operated by the Alliance for Sustainable Energy, LLC
A Strong Energy Portfolio for a Strong America

Energy efficiency and clean, renewable energy will mean a stronger economy, a cleaner environment, and greater energy independence for America. Working with a wide array of state, community, industry, and university partners, the U.S. Department of Energy's Office of Energy Efficiency and Renewable Energy invests in a diverse portfolio of energy technologies.

DOE/GO-102009-2757 • April 2009

Printed with a renewable-source ink on paper containing at least 50\% wastepaper, including $10 \%$ postconsumer waste. 